\title{
Efficacy of Bacteriophage Cocktails on E. coli K-12 in HEK Cells
}

\author{
Katherine D. Le \\ Palos Verdes Peninsula High School, 27118 Silver Spur Rd, Rolling Hills Estates, CA, 90274, U.S.A.; katherinedle@gmail.com
}

\begin{abstract}
Bacteriophages are viruses that infects specific targeted bacteria, reproducing within it. The therapeutic use of phages to treat bacterial infections is known as phage therapy. Phage therapy has the potential to treat infections and could be an alternative to antibiotics, especially against antibiotics resistance strains. Bacteriophage (phage) synergy refers to a phenomenon where the antibacterial properties of phages are augmented when two or more phages are combined. It was hypothesized that due to phage synergy, a T2+T4 phage cocktail would exhibit enhanced antimicrobial properties against $E$. coli K-12 and would demonstrate therapeutic properties in presence of HEK cells. Through measuring the combinational effects of the two phages on the bacteria, it was tested to observe whether the phages individually and together were toxic to the human cells. Plaque assays were performed to quantify the amount of plaque forming units (PFU) in the phage samples. In the plaque assays, the phage cocktail was not as effective against the E. coli K-12 compared to individual phages. However, for the cell culture assays, the cocktail was observed to exhibit increased effectivity. The data also indicated that the phages were not lethal to the HEK cells. The infection and lysis of the bacteria also did not have any detrimental health effects towards the human cells. Phage therapy holds the potential to provides a cost-efficient method of treatment and can be an alternative to antibiotics resistance strain of bacteria.

KEYWORDS: Health; microbiology; bacteriophage; phage synergy; HEK cells; plaque assays; antibiotics; antibiotic resistance.
\end{abstract}

\section{- Introduction}

Bacteriophages are viruses that can infect specific bacteria. Bacteriophage cocktails contain a mixture of two or more phages and are believed to have enhanced antimicrobial effects. Bacteriophages infects and kills bacteria by causing the cell to burst or lyse. Through injecting its genes into the bacteria, the phage is able to reproduce. The reproduction of phages within the host cell allows more phages to be released, going off to kill more of the bacteria. Phages carry a unique characteristic of being self-dosing and self-limiting. This means that bacteriophages have the ability to limit itself once there is no more host cell. The phage will go away on its own without any need of treatment or medication.

Bacteria have become increasingly resistant to antibiotics making bacterial illnesses to be harder to treat. Antibiotic resistance is responsible for a rise in multiple deadly illnesses including but not limited to pneumonia and tuberculosis. Antibiotic resistance is a global crisis, threatening not only health, but also agriculture, causing food security and increased medical costs. Because of their unique ability to only infect specific types of bacteria without harming human cells, bacteriophages have been proposed as an alternative and sustainable antimicrobial treatment to address the issue of antibiotic resistance.

The approach of using phages to treat diseases is known as phage therapy. "Bacteriophages could in theory be used in isolation or combination when a single phage is not sufficient to treat a bacterial disease" ${ }^{1}$ Cocktails containing multiple phages are expected to offer enhanced bacterial effects due to a phenomenon known as phage synergy, where the viruses work synergistically to infect and kill bacteria. The phenomenon of synergy is complex and has not been studied often. It was found that this phenomenon is caused by the activity of a phage's tailspike and the enzymes it contains. "A biological context for this augmentation is tailspike enzymes. Phage tailspikes commonly carry enzymes that degrade extracellular carbohydrates produced by bacteria, enhancing access to the cell surface" 2 Phage synergy therefore increases and speeds up the process of lysis causing more effective eradication of a target bacterium.

Creating new antibiotics is very costly-pharmaceutical companies are reluctant to take it on because resistance develops so quickly, they can't get a good return on their investment. Phages offer an attractive alternative to new antibiotic develop because they are the natural predators to bacteria and able to evolve to overcome their hosts defenses. They are also inexpensive to produce, safe for human consumption and sufficiently abundant to target a wide range of bacterial pathogens.

This project tests the potential of using combinatorial phage cocktails as a therapy on human embryonic kidney cells, or HEK cells. Phages T2 and T4 are used for the cocktail due to them being in the same family therefore having similar properties to increase phage synergy. The cocktail is introduced to the cells, and their effectiveness against $E$. coli is observed as well as their effect on the HEK cells. Phage T2 and T4 will also be tested separately against the $E$. coli and their individual efficacy compared against the cocktail.

Would a combination of phages be more effective against a target bacterium in HEK cells compared to one phage type working alone? Combining multiple phages from the same subgroup is hypothesized to more effectively kill the targeted bacteria compared to a singular phage type working alone and antibiotics because of the phenomenon of phage synergy where 
one phage augments the properties of another in a cocktail of phages. Studying and experimenting with the interactions within a mixture of phages could broaden phage therapy and possibly strengthen phages as therapeutic agents.

\section{Methods}

\section{Bacterial Culture:}

E. coli was grown in $\mathrm{LB}$ broth overnight at $37^{\circ} \mathrm{C}$ in a shaking incubator to an OD600 of 0.5. An OD600 reading of the $E$. coli was be done to ensure that the $E$. coli is in a good state to form a nice even lawn. An OD600 reading is an optical density reading at 600 nanometers using a spectrophotometer. Performing an OD600 reading estimates the concentration of the cells. An OD600 reading from the range of $0.5-0.7$ is ideal.

\section{Phage dilutions :}

Bacteriophages T2 and T4 were purchased from Carolina Biological and diluted to the required titer by ten-fold serial dilutions in $100 \mu \mathrm{l}$ of LB broth $(1 \% \mathrm{w} / \mathrm{v}$ solution dissolved in water). Phage assays were performed using $10 \mu \mathrm{l}$ of diluted phage preparations.

\section{Plaque Assay:}

Plaque assays ${ }^{3}$ were performed in 12 -well culture plates using the agar overlay method. ${ }^{4}$ Performing this assay results in plaque forming units (PFU) which are small holes that indicate where the bacteria have been killed. Assay plates were poured with $1 \mathrm{ml}$ per well of LB-bottom agar $(1 \% \mathrm{w} / \mathrm{v}$ solution dissolved in water). Soft top-LB agar overlay ( $1 \% \mathrm{w} / \mathrm{v}$ solution dissolved in water) was stored at $4^{\circ} \mathrm{C}$ and warmed to $45^{\circ} \mathrm{C}$ prior to use. Phage assays were performed by preparing a bacterial overlay solution containing $0.5 \mathrm{ml}$ of E.coli and $4.25 \mathrm{ml}$ of soft top LB-agar (sufficient concentration to produce a bacterial lawn). For each well, $300 \mu \mathrm{l}$ of the bacterial overlay solution was inoculated with $10 \mu \mathrm{l}$ of diluted phage and carefully pipette into the well, over the LB-Bottom agar. The soft agar tops were allowed to set at room temperature for 15 minutes before the plate was transferred to a $37^{\circ} \mathrm{C}$ incubator overnight. Plaques were visible the next days as holes in the surrounding bacterial lawn. Control wells consisted of untreated bacterial lawns. All experiments were carried out in triplicate with three technical replicates per assay plate.

\section{Data Analysis:}

Using the equation: PFU/ $\mu \mathrm{l}=$ Number of Plaques Counted/ (Dilution x Volume of Diluted Virus Added) ${ }^{5}$ we were able to calculate the amount of plaque forming units per microliter for each phage individually and combined.

\section{Human Cell Culture:}

HEK cells were cultured on cell culture treated plastic in a growth medium containing DMEM supplemented with 10\% FBS and $1 \mathrm{mM}$ L-Glutamine. Cells were maintained at $37^{\circ} \mathrm{C}$ in a humidified incubator in an atmosphere containing $5 \%$ $\mathrm{CO}_{2}$. Confluent cells were passaged $1: 4$ at by standard trypsin passaging twice per week.

\section{HEK Phage Assays:}

HEK cells were trypsin dissociated, counted, and seeded into 24 well plates at a density of $3 \times 10^{5}$ cells per well. Cells were treated 12 hours after plating with undiluted phage (T4 (2.2x10 ${ }^{9}$ PFU) $10 \mu \mathrm{l}$; T2 (7.3×10 ${ }^{9}$ PFU) $10 \mu \mathrm{l}$; T4+T2 cocktail $10 \mu \mathrm{l}$ of each phage) and $1 \mu \mathrm{l}$ of E.coli (cultured to an OD600 of 0.5 ) for 24 hours at $37^{\circ} \mathrm{C}$. Image analysis of HEK cell viability was performed using a phase contrast microscope. Bacterial growth was assessed by measuring the optical density (OD) of the cell culture medium at $600 \mathrm{~nm}$ using an absorbance plate reader.

\section{Analyzing HEK Cells:}

The HEK cells are to be visually analyzed and interpreted. The phage's effectiveness can be seen when looking at the amount of $E$. coli that was eradicated.

To further analyze the effectiveness of the phages, OD600 readings were done for the remaining $E$. coli after the assays.

\section{- Results}

Phage Cocktails composed of T2 and T4 bacteriophage did not display enhanced antimicrobial activity during soft overlay

\section{plaque assays:}

We sought to determine whether treatment of bacteria with a combination of T2 and T4 would exhibit enhanced antibacterial properties during standard plaque assays. It was observed that the combination of phages did not eradicate as much bacteria compared to T2. Phage T2 alone showed the most effectiveness against the $E$. coli $\mathrm{K}-12$ and phage $\mathrm{T} 4$ alone displaying the least effectiveness against the bacteria. Table 1 shows the amount of plaque forming units per $\mu \mathrm{l}$ in the three trials of the standard plaque assays. The cocktail of phage T2 and T4 did not appear to have enhanced antibacterial properties. Therefore, the phenomenon of phage synergy did not appear to be present.

Treatment of HEK cells with T2 and T4 bacteriophages did not cause adverse effects to cell viability or growth:

To confirm T2 and T4 bacteriophages do not affect the human cells in any negative way, the phages were added with the HEK cells alone. Figure 3 shows the cells when exposed to the phages. The HEK cells did not display any adverse effects or abnormalities in health or growth when the phages were introduced. The phages did not affect the HEK cells in any negative way, which displays the phages' therapeutic properties.

A combined cocktail of T2 and T4 bacteriophages demonstrated enhanced antimicrobial properties during HEK cell culture assays:

Having determined that T2 and T4 bacteriophages do not adversely affect the general health, growth, or survival of HEK cells, we next sought to determine whether combinatorial phage therapy could be used to eliminate $E$. coli when in the presence of HEK cells. It was observed that both phages T2 and T4 individually failed to eliminate the majority of the bacteria. The combination of phages appeared to eliminate nearly all of the bacteria. The phage cocktail showed to be more effective against the bacteria and therefore displayed the phenomenon of phage synergy and phage therapy.

\section{HEK cells when exposed to the E. coli alone:}

A control was done with the HEK cells and the E. coli K-12 alone. It was observed that the bacteria invaded the space of 
the HEK cells, therefore limiting its area to grow. The phages having eliminated most of the bacteria, allowed the cells to grow more effectively. Figure 4 shows a graph of $E$. coli growth in HEK cell cultures following treatment with $\mathrm{T} 2$ and T4 bacteriophages.

\section{- Discussion \\ Bacteriophage cocktail in the plaque assay did not demonstrate antimicrobial properties:}

In the plaque assays, the combination of phages did not display strong antimicrobial properties. In the plaque assays, the hypothesis was shown to be incorrect since phage T2 formed a greater amount of plaques compared to the cocktail in all three trials.

\section{Plaque assays did not exhibit the phenomenon of phage} synergy:

Phage synergy did not appear to be strongly present in the plaque assays, with T2 exhibiting more plaques compared to the cocktail. T2 eradicated so much of the bacteria that it made it difficult to obtain an average of plaque forming units. The average plaque forming units was $5.3 \times 10^{9} \mathrm{PFU} / \mu \mathrm{l}$ for the phage cocktail and $1.5 \times 10^{9} \mathrm{PFU} / \mu \mathrm{l}$ for phage T4. A more diluted form of phage T2 could have allowed the formation of clearer plaques instead of total destruction. Using a different ratio of T2:T4 also could have affected the results of the phage cocktail. Adding a greater amount of T2 could possibly allow the combination of phages to enhance their performance in the standard plaque assays.

\section{Bacteriophage cocktail in human cells did not harm the cells:}

The initial test with the HEK cells exposed to the phage alone showed that the phage did not affect the HEK cells in any harmful or abnormal way. After careful observation, it was concluded that in the presence of the HEK cells, the idea of phage therapy was able to be carried out. The cells remained healthy as the phages and the phage cocktail eradicated the E. coli.

\section{In the culture assays, the phage cocktail displays phage} synergy:

Table 1 : Table of the three trials of the plaque assays and each trial's average $\mathrm{PFU} / \mu \mathrm{l}$ for phages individually and combined.

\begin{tabular}{|l|c|c|c|}
\hline & Trial 1 & Trial 2 & Trial 3 \\
\hline T2 & $1.22 \times 10^{10} \mathrm{PFU} / \mu 1$ & $\begin{array}{c}\text { too many plaques to be } \\
\text { quantifiable }\end{array}$ & $\begin{array}{c}\text { too many plaques to be } \\
\text { quantifiable }\end{array}$ \\
\hline $\mathbf{T 4}$ & $6.8 \times 10^{9} \mathrm{PFU} / \mu 1$ & $7.0 \times 10^{8} \mathrm{PFU} / \mu 1$ & $6.0 \times 10^{9} \mathrm{PFU} / \mu 1$ \\
\hline $\mathbf{T 2}+\mathbf{T 4}$ & $3.9 \times 10^{9} \mathrm{PFU} / \mu 1$ & $2.27 \times 10^{10} \mathrm{PFU} / \mu 1$ & $2.11 \times 10^{10} \mathrm{PFU} / \mu 1$ \\
\hline
\end{tabular}

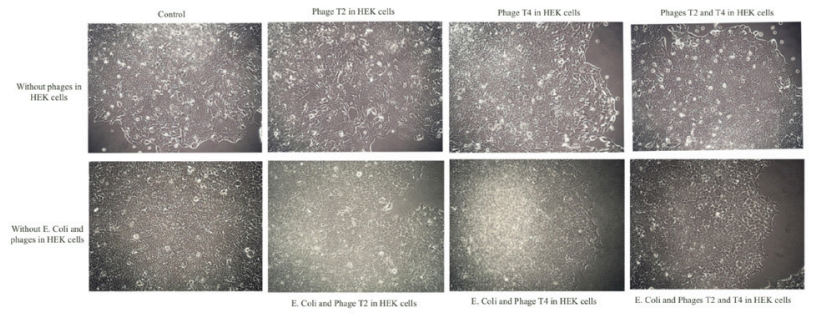

Figure 3 : Upper row contains images of HEK cell controls and with phages individually and combined. Bottom row contains images of HEK cells with $E$. coli K-12 and phages individually and combined.

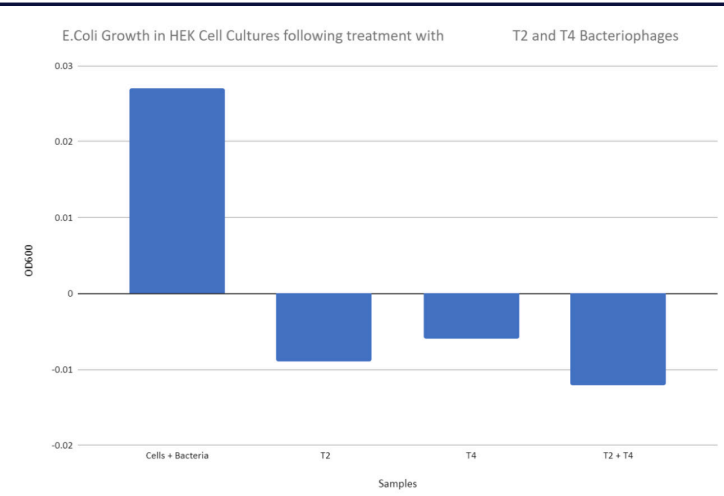

Figure 4 : Graph of OD600 readings based on E. coli growth in HEK cell cultures following treatment with $\mathrm{T} 2$ and $\mathrm{T} 4$ bacteriophages.

Table 2 : Table containing the OD600 readings corelating to the graph of E. coli growth in HEK cell cultures following treatment with T2 and T4 bacteriophages.

\begin{tabular}{|l|c|c|c|c|c|}
\hline & $\mathbf{1}$ & $\mathbf{2}$ & $\mathbf{3}$ & Average & SD \\
\hline Cells + Bacteria & 0.01 & $\mathbf{0 . 0 0 9}$ & 0.008 & 0.027 & 0.001 \\
\hline T2 & -0.005 & $\mathbf{0 . 0 0 2}$ & -0.006 & -0.009 & 0.004 \\
\hline T4 & -0.003 & -0.001 & -0.002 & -0.006 & 0.001 \\
\hline T2 + T4 & -0.006 & -0.005 & -0.001 & -0.012 & 0.003 \\
\hline
\end{tabular}

In the experimentation with the HEK cells, phage synergy of the cocktail was observed to have eradicated more bacteria compared to both of the phages separately. According to the "Synergy as a rationale for phage therapy using phage cocktails" study: "Synergy alters the dynamics by increasing the growth rate of one phage in the presence of the other. This interdependence between the phages is the key to the maintenance of both, but the interdependence alone does not ensure that both phages are maintained." ${ }^{1}$

\section{The phage cocktail demonstrates therapeutic properties:}

Results from the human cell culture assays supports the initial hypothesis that due to phage synergy, a T2+T4 phage cocktail would exhibit enhanced antimicrobial properties against $E$. coli K-12 and would demonstrate therapeutic properties in presence of HEK cells. Referring to Figure 6 and 7, it was shown that with the combination of phages, the average OD600 reading of the remaining bacteria is -0.012 . This negative absorbance indicates that the concentration of bacteria present is very low. Compared to phage T2 with an average OD600 reading of -0.009 and phage $\mathrm{T} 4$ with -0.009 , the cocktail of phages performed more effectively.

\section{Potential for combined phage therapy:}

This experiment tested bacteriophages and its possibility to act as a therapy. The data demonstrated that phage synergy is present, although appears to be limited in effectivity. Bacteriophage cocktails also displayed its potential by appearing to be safe to human cells while still eliminating the target bacteria. Factors that could improve the phenomenon of phage synergy includes having more phages from the Tevenvirinae family that could have been added to potentially increase the phage synergy. Another improvement to phage synergy could be done by choosing a better combination of phages.

Further research would include using the modern technology of genetic modification to modify a phage to allow it to become more effective as a phage therapy. Technology such 
on phage therapy could also be done to further investigate how phage therapy can be developed and used for bacteria derived illnesses such as tuberculosis and pneumonia.

The use of bacteriophages as a therapy, holds the potential to reduce the rising issue of antibiotic resistance while also providing a cost-efficient method of treatment.

\section{Acknowledgements}

Thank you to Dr. Victoria Fox and George Tseng at Pathways to Stem Cell Science for mentoring and helping me with my project.

\section{- References}

1. Schmerer, M., Molineux, I. J., \& Bull, J. J. (2014). Synergy as a rationale for phage therapy using phage cocktails. PeerJ, 2, e590. https://doi.org/10.7717/peerj.590.

2. Bull \& Molineux (2008) Bull JJ, Molineux IJ.

Predicting evolution from genomics: experimental evolution of bacteriophage t7. Heredity. 2008;100(5): 453-463. doi: 10.1038/sj.hdy.6801087.

3. JoVE Science Education. (n.d.). Plaque Assay: A method to Determine Viral TITER as PLAQUE Forming Units (PFU). https://www.jove.com/v/10514/plaque-assay-methodto-determine-viral-titer-as-plaque-forming-units .

4. David Fankhauser. (2016, May 15). Agar overlay technique. https://fankhauserblog.wordpress.com/1994/05/ 04/agar-overlay-technique/\#: :text=This\%20tech nique\%20allows\%20you\%20to,cooled $\% 20$ to $\% 2045 \% \mathrm{C} 2 \% \mathrm{~B} 0 \mathrm{C}$.

5. Lee, A. (2020, April 15). Plaque assayin 1 collection. https://www.protocols.io/view/plaque-assaybe6sjhee.html\#: :text=Abstract,concentration $\% 20$ of\% 20a\%20given\%20sample.

6. Bacteriophage.news. (2020, December 28).

Bacteriophage preparation methodology for phage cocktails and products. https://www.bacteriophage.news/bacteriophagepreparation-methodology-for-phage-cocktails-andproducts/.

7. Casey, E., van Sinderen, D., \& Mahony, J. (2018).

In Vitro Characteristics of Phages to Guide

'Real Life' Phage Therapy Suitability. Viruses, 10(4), 163. https://doi.org/10.3390/v10040163.

\section{- Author}

Katherine Le is currently a sophomore at Palos Verdes Peninsula High School who thoroughly enjoys the field of math and science. She recently discovered her passion for microbiology in her freshman year of high school, participating in the science fair. Being the secretary of the math team and science research club, she further expanded her knowledge and interest of science. In the future she hopes to work more with microbiology and pursue a career in the medical field. 